\title{
Transfer-matrix approach based on modal analysis for modeling corrugated long-period fiber gratings
}

\author{
Gia-Wei Chern, Lon A. Wang, and Chunn-Yenn Lin
}

\begin{abstract}
A transfer-matrix method is developed for modeling a corrugated long-period fiber grating. Claddingmode resonance in such a corrugated structure can be controlled by the applied tensile stress based on the photoelastic effect. A first-order vectorial perturbation expansion is used to derive the mode fields of the two basic regions under the strain-induced index perturbation. Because the etched cladding radius is much smaller than the unetched radius, the effect of the corrugated structure on cladding modes cannot be treated as a small perturbation. Thus the conventional coupled-mode theory is inadequate for the modeling of such a structure. Based on a self-consistent mode-matching technique, mode coupling within the corrugated structure can be described by a set of transfer matrices. We apply the formulation to the calculation of the transmission spectra of a corrugated long-period grating and compare the calculated with the experimental results. The transfer-matrix approach is found to account well for the features of the transmission spectra of the corrugated long-period gratings. (C) 2001 Optical Society of America

OCIS codes: $\quad 050.2770,060.2280,060.2340$.
\end{abstract}

\section{Introduction}

The long-period fiber grating (LPFG) has become an important component of optical filters, ${ }^{1}$ gain equalizers, ${ }^{2}$ polarizers, ${ }^{3}$ and sensors. ${ }^{4,5}$ Under phasematching conditions a LPFG can couple light from the fundamental core mode to a specific cladding mode and thus contribute to a loss dip in the transmission spectrum. Conventional LPFG's fabricated by exposure of photosensitive fibers to ultraviolet light have little tunability on the peak losses and resonant wavelengths. It has been reported that high tuning efficiency of the resonant wavelength can be realized by electrical heating of a metal-coated air-clad LPFG. ${ }^{6}$ Based on the acousto-optic effect, a loss-tunable LPFG can also be made by application of an acoustical wave. ${ }^{7}$ Lin and Wang 8 proposed a novel corrugated structure for forming a loss-tunable LPFG based on the photoelastic effect. The peak loss of the corrugated LPFG can be adjusted by an applied tensile force, and the resonant wavelength is insensitive to tensile stress. The corrugated struc-

The authors are with the Department of Electrical Engineering and Institute of Electro-Optical Engineering, National Taiwan University, Taipei, Taiwan. L. A. Wang's e-mail address is lon@ccms.ntu.edu.tw.

Received 17 November 2000.

0003-6935/01/254476-11\$15.00/0

(C) 2001 Optical Society of America ture is shown schematically in Fig. 1. It consists of two basic regions, unetched and etched. Inasmuch as the strains induced within the etched and the unetched regions are inversely proportional to the corresponding cross-sectional areas under mechanical equilibrium, a long-periodic index modulation is produced as a result of the photoelastic effect and the periodic strain distribution.

Conventionally, the couplings among core and cladding modes caused by LPFGs can be described well by the coupled-mode theory. ${ }^{9}$ In such a formulation the grating is treated as a perturbation of the original fiber waveguide, and couplings among the modes are described by a set of ordinary differential equations. Because the cladding mode is guided by the claddingair interface, for the corrugated LPFG the cladding modes of the two basic regions cannot be treated perturbatively because there is a large difference in their cladding radii. The coupled-mode theory is then inapplicable in our case. In this paper we propose an approach based on a mode-matching technique and a transfer-matrix method to describe the interactions of core and cladding modes within the corrugated LPFG. The transfer-matrix formulation is similar to that proposed in Ref. 10 for a photoinduced binary LPFG. In the case of a corrugated LPFG the mode fields of the two basic waveguides are solved independently by the finite-difference method. These mode fields are regarded as unperturbed with respect to strain-induced index perturbation, and we 

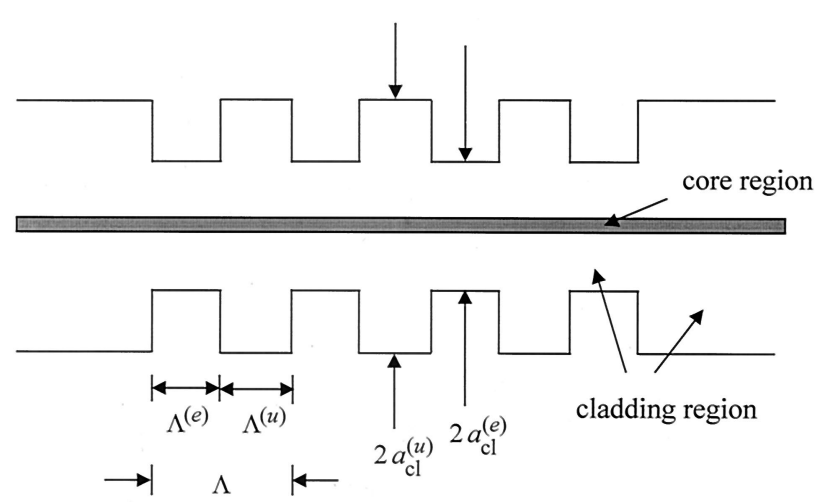

Fig. 1. Schematic diagram of a corrugated LPFG.

use first-order vectorial perturbations ${ }^{10}$ to obtain the mode fields and the corresponding propagation constants under applied tensile stress. At the interfaces of the two regions, the mode-matching technique is then used to yield coupling of mode amplitudes as a result of discontinuities of the guiding structure. Basically, we expand the electric and magnetic fields of the two guides by using the corresponding complete set of mode fields and by imposing the continuity conditions on the interface. With appropriate approximations, we can use a forward transfer-matrix method to describe the couplings of the heterointerfaces.

The remainder of this paper is organized as follows: In Section 2 we apply first-order perturbation expansions to the mode fields of the two basic regions under strain-induced index perturbation. We then introduce a self-consistent mode-matching technique in Section 3 with which we derive the scattering coefficients of the junctions formed by the etched and unetched regions. The contributions from the backward-propagating mode amplitudes are found to be much smaller than those from the transmitted amplitudes. Thus, by neglecting these backwardpropagating modes, in Section 4 we derive a set of forward transfer matrices to describe the evolution of mode amplitudes. To illustrate the features of the method, we consider only couplings from the fundamental core mode to the first cladding mode. The numerical calculation of a specific example of a LPFG is described in Section 5. The results are compared with the measured spectra, and an overall agreement is obtained. We present our conclusions in Section 6.

\section{First-Order Expansion of Mode Fields under Strain-Induced Index Perturbation}

The corrugated LPFG is composed of two basic concatenating waveguides, to which we refer to as guide $(u)$ and guide (e), which correspond to unetched and etched regions, respectively, as shown in Fig. 1. We fabricated the corrugated structure by periodically etching the cladding radius of an optical fiber, thus forming a section with periodic variation of crosssectional areas within the fiber. The lengths of the unetched and etched regions within a single period

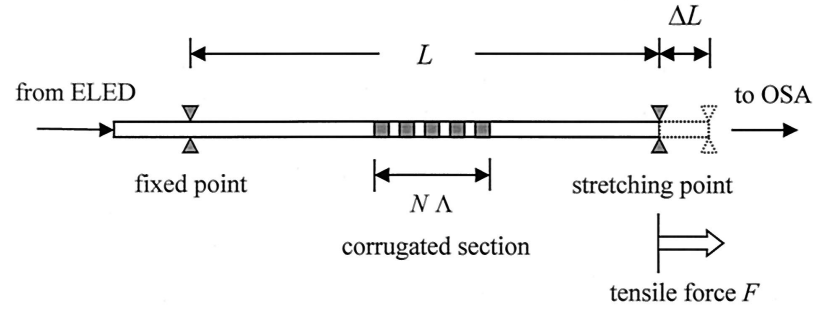

Fig. 2. Experimental setup for applying tensile force and measuring the transmission spectra of a corrugated LPFG: ELED, electroluminescent (light-emitting) diode; OSA, optical spectrum analyzer.

are $\Lambda^{(u)}$ and $\Lambda^{(e)}$, respectively; thus the periodicity of the corrugated structure is $\Lambda=\Lambda^{(u)}+\Lambda^{(e)}$. The following convention is used throughout this paper: Quantities that correspond to the unetched region are represented by symbols with superscripts $(u)$; those of the etched region, with a superscripts $(e)$. The cladding radii of the unetched and etched regions are $a_{\mathrm{cl}}^{(u)}$ and $a_{\mathrm{cl}}^{(e)}$, and the radial index profiles are $n_{0}^{(u)}(r)$ and $n_{0}^{(e)}(r)$, respectively. The $z$ axis is taken along the fiber axis, and a tensile force $F$ is applied along the fiber as shown in Fig. 2. The corrugated section lies within the central region of a fiber of total length $L$; one end of the fiber is fixed, while the other end is stretched along the fiber axis to produce a tensile strain by applied force $F$. The longitudinal displacement of the stretching point is $\Delta L$; thus the induced tensile strain of the fiber is $s_{z}=\Delta L / L$.

In the following discussion we neglect the effect of stress concentration at the discontinuities of the two regions by assuming that the sharp discontinuity is a fairly narrow smooth transition region. As mechanical equilibrium is established, the longitudinal forces become increasingly similar for the etched and unetched regions, and both are equal to applied force $F$. The etched and unetched regions will thus experience different tensile strains, which are inversely proportional to the cross-sectional areas of the corresponding regions. ${ }^{11}$ The induced tensile strains $s_{z}^{(u)}$ and $s_{z}^{(e)}$ in the two regions are

$$
\begin{gathered}
s_{z}^{(u)}=\frac{F}{Y A_{\mathrm{cl}}^{(u)}} \cong \frac{\Delta L}{L}=s_{z}, \\
s_{z}^{(e)}=\frac{F}{Y A_{\mathrm{cl}}^{(e)}}=\left[\frac{a_{\mathrm{cl}}^{(u)}}{a_{\mathrm{cl}}^{(e)}}\right]^{2} s_{z},
\end{gathered}
$$

where $Y$ is Young's modulus of the fiber and $A_{\mathrm{cl}}^{(u)}$ and $A_{\mathrm{cl}}^{(e)}$ are areas of cladding of the unetched and etched regions, respectively. In Eq. (1a) we have approximated the tensile strain of unetched region $s_{z}$ by the overall measured strain $\Delta L / L$. Doing so is valid because the length of the corrugated section is negligible compared with the total length of fiber that is subject to force $F$ : i.e., $N \Lambda \ll L$, where $N$ is the number of periods. This condition is usually satisfied in our experiments. From Eq. (1) we can see that, when a tensile force is applied, a periodic strain distribution is induced within the corrugated section. 
And, from Eq. (1b), the strain difference increases as the ratio of the cladding radii increases. The transverse strain is related to the tensile strain by Poisson's ratio $v$ as $s_{t}=-v s_{z}$, and, through the photoelastic effect, the strain-induced index perturbations of the two regions $\operatorname{are}^{12}(r=u$ or $r=e)$

$$
\begin{aligned}
\Delta n_{t}^{(r)} & =-1 / 2\left(p_{12}-v p_{11}-v p_{12}\right)\left[n_{0}^{(r)}\right]^{3} s_{z}^{(r)} \\
& =-1 / 2 p_{t}\left[n_{0}^{(r)}\right]^{3} s_{z}^{(r)} \\
\Delta n_{z}^{(r)} & =-1 / 2\left(p_{11}-2 v p_{12}\right)\left[n_{0}^{(r)}\right]^{3} s_{z}^{(r)} \\
& =-1 / 2 p_{z}\left[n_{0}^{(r)}\right]^{3} s_{z}^{(r)}
\end{aligned}
$$

where $p_{11}$ and $p_{12}$ are the photoelastic constants ${ }^{12}$ and, for simplicity, we have introduced two effective photoelastic coefficients, $p_{t}$ and $p_{z}$. Note that the waveguides under tensile strain become slightly anisotropic with uniaxial symmetry. These index variations will introduce perturbations to the field distribution and the propagation constant of the waveguide modes.

We use a vectorial perturbation expansion to find the corrections of these modes to first order in strain. The vectorial perturbation of mode fields is based mainly on the reciprocity formulas ${ }^{13}$ that connect the unperturbed mode fields and the perturbed fields. In Appendix A we derive the reciprocity formulas for anisotropic waveguides.

Using the notation of Appendix A, let the primed fields be the unperturbed mode fields and the unprimed fields be the mode fields under straininduced index perturbation. By iterative substitution for the reciprocity formulas [Eqs. (A9) and (A12) below], one can derive an expansion of the mode fields with respect to the small perturbation parameter, $s_{z}$. In what follows, we use symbols with overbars to represent unperturbed mode fields in the two regions. Thus the unperturbed fields of mode $k$ of guide $r$ are denoted $\left[\overline{\mathbf{e}}_{k}^{(r)}, \overline{\mathbf{h}}_{k}^{(r)}\right]$. The corresponding mode fields under strain perturbation are $\left[\mathbf{e}_{k}^{(r)}, \mathbf{h}_{k}^{(r)}\right]$. All these mode fields are orthogonal and normalized to carry unity power; i.e.,

$$
1 / 2 \int_{A_{\infty}}\left[\mathbf{e}_{k}^{(r)} \times \mathbf{h}_{j}^{(r) *}\right] \cdot \mathbf{z} \mathrm{d} A=\delta_{k j},
$$

where $\delta_{k j}$ is the Kronecker delta symbol.

Similar expressions hold for the unperturbed mode fields. Because the fiber used in our experiment is weakly guiding, the $z$ component of the mode fields is negligible compared with that of the transverse fields, so the mode fields can be regarded as linearly polarized fields. Thus, in what follows, we neglect the contributions from the $z$ component. For clarity, the superscripts $(u)$ and $(e)$ will not be shown in the following discussion, because the formalism can be applied to both guides.
First we expand the perturbed mode fields by using the complete set of unperturbed fields as follows:

$$
\begin{aligned}
\mathbf{e}_{t j} & =\sum_{k}\left(a_{j k}+b_{j k}\right) \overline{\mathbf{e}}_{t k}, \\
\mathbf{h}_{t j} & =\sum_{k}\left(a_{j k}-b_{j k}\right) \overline{\mathbf{h}}_{t k} .
\end{aligned}
$$

The expansion coefficients are given by the reciprocity formula [Eqs. (A12) below]. For a first-order perturbation, when we approximate the perturbed quantities by the corresponding unperturbed quantities and neglect contributions from $z$ components of the mode fields, the following results are obtained $(j \neq k)$ :

$$
\begin{aligned}
a_{j k} & =\frac{k_{0}}{4\left(\bar{\beta}_{j}-\bar{\beta}_{k}\right)} \frac{1}{Z_{0}} \int_{A_{\infty}} 2 n_{0} \Delta n_{t} \overline{\mathbf{e}}_{t j} \cdot \overline{\mathbf{e}}_{t k} * \mathrm{~d} A, \\
b_{j k} & =\frac{k_{0}}{4\left(\bar{\beta}_{j}+\bar{\beta}_{k}\right)} \frac{1}{Z_{0}} \int_{A_{\infty}} 2 n_{0} \Delta n_{t} \overline{\mathbf{e}}_{t j} \cdot \overline{\mathbf{e}}_{t k} * \mathrm{~d} A .
\end{aligned}
$$

In the above expressions we have used the approximation $n_{t}^{2}-n_{0}^{2} \cong 2 n_{0} \Delta n_{t}$, with $\Delta n_{t}$ given by Eq. (2). For the corrections of the propagation constants we use Eq. (A9) below, and for first-order perturbation we have

$$
\beta_{j}-\bar{\beta}_{j}=\frac{k_{0}}{4 Z_{0}} \int_{A_{\infty}} 2 n_{0} \Delta n_{t} \mathbf{e}_{t j} \cdot \overline{\mathbf{e}}_{t j}^{*} \mathrm{~d} A .
$$

Because in our case the optical power is coupled from the core mode to the nearly phase-matched cladding mode, the propagation constants of these modes are extremely similar. And, inspecting Eqs. (6) in which the propagation constants are subtracted from the denominator of $a_{j k}$ while they are added to it in $b_{j k}$, we expect that $a_{j k} \gg b_{j k}$ for the coupled modes and neglect the contributions of $b_{j k}$ in what follows. By renormalizing the derived perturbed mode fields ${ }^{10}$ we obtain the following results:

$$
\begin{gathered}
\mathbf{e}_{j} \cong\left(1-d_{j} s_{z}^{2}\right) \overline{\mathbf{e}}_{j}-s_{z} \sum_{k \neq j} \frac{\kappa_{j k}}{\left(\bar{\beta}_{j}-\bar{\beta}_{k}\right)} \overline{\mathbf{e}}_{k}, \\
\mathbf{h}_{j} \cong\left(1-d_{j} s_{z}^{2}\right) \overline{\mathbf{h}}_{j}-s_{z} \sum_{k \neq j} \frac{\kappa_{j k}}{\left(\bar{\beta}_{j}-\bar{\beta}_{k}\right)} \overline{\mathbf{h}}_{k},
\end{gathered}
$$

where, from Eqs. (6) and (8), $\kappa_{j k}$, given by

$$
\kappa_{j k}=\frac{p_{t} \omega \varepsilon_{0}}{4} \int_{A_{\infty}}\left[n_{0}(r)\right]^{4} \overline{\mathbf{e}}_{t j} \cdot \overline{\mathbf{e}}_{t k} * \mathrm{~d} A,
$$

is the intrinsic cross-coupling constant between mode $j$ and mode $k$ as a result of the photoelastic effect; $d_{j}$ is introduced here to ensure that the perturbed mode fields are also normalized to carry unity power. ${ }^{10}$ The modified propagation constant can be found from Eq. (7) as

$$
\begin{aligned}
\beta_{j} & =\bar{\beta}_{j}+s_{z} \Delta \beta_{j} \\
& =\bar{\beta}_{j}-s_{z} \kappa_{j j},
\end{aligned}
$$


where $\kappa_{j j}$ is the self-coupling constant given by Eq. (9) with $j=k$ :

$$
\kappa_{j j}=\frac{p_{t} \omega \varepsilon_{0}}{4} \int_{A_{\infty}}\left[n_{0}(r)\right]^{4}\left|\overline{\mathbf{e}}_{t j}\right|^{2} \mathrm{~d} A .
$$

\section{Scattering Coefficients for Hetero-Interfaces of the Corrugated Structure}

Conventionally, coupled-mode theory is used to describe the interactions between waveguide modes when there is a longitudinal perturbation within the guide. However, in the case of a corrugated LPFG the etching depth is so large that we cannot regard the corrugated structure as a perturbation. A rigorous analysis for mode coupling within the corrugated LPFG cannot be based on coupled-mode theory, and treating such discontinuities will require other approaches.

Because we want to know how the optical power is transferred between waveguide modes, methods based on modal analysis are more efficient in this respect. ${ }^{14,15}$ In these approaches the number of modes is truncated to a prescribed integer, and we can use the continuity conditions of the electromagnetic fields to obtain a set of linear equations for solving the scattering coefficients of the discontinuous interfaces.

In this section we use a self-consistent modematching technique to find the scattering coefficients of the heterointerfaces between the unetched and the etched regions. The approach described below is based mainly on the arguments of Ref. 16, in which the reflected amplitudes are assumed to be negligible compared with the transmitted amplitudes, and approximate formulas are derived for these scattering coefficients. The results are self-consistent in the sense that the derived reflection coefficients are indeed much smaller than the transmission coefficients. In addition, from the experiment viewpoint the measured reflected power is indeed negligible.

Now consider the situation depicted in Fig. 3(a), where a wave field of mode $k$ in the unetched region is incident upon the heterojunction. This wave field will partially transmit with coefficient $t_{k k}$ and partially reflect with coefficient $r_{k k}$. In addition, it will also excite other forward- and backward-traveling modes with cross-transmission and -reflection coefficients $t_{k j}$ and $r_{k j}$, respectively, for mode $j$. These scattering coefficients are also shown schematically in the figure. The corresponding primed coefficients, $t_{k j}^{\prime}$ and $r_{k j}^{\prime}$, refer to the scattering coefficients for wave fields incident from the etched region to the unetched region, as shown in Fig. 3(b). Let the amplitude of the incident mode be $A$; then the continuity conditions of the electric and magnetic fields require that

$$
\begin{aligned}
& \left(A+A r_{k k}\right) \mathbf{e}_{t k}^{(u)}+\sum_{j \neq k} A r_{k j} \mathbf{e}_{t j}^{(u)}=\sum_{j} A t_{k j} \mathbf{e}_{t j}^{(e)}, \\
& \left(A-A r_{k k}\right) \mathbf{h}_{t k}^{(u)}-\sum_{j \neq k} A r_{k j} \mathbf{h}_{t j}^{(u)}=\sum_{j} A t_{k j} \mathbf{h}_{t j}^{(e)} .
\end{aligned}
$$

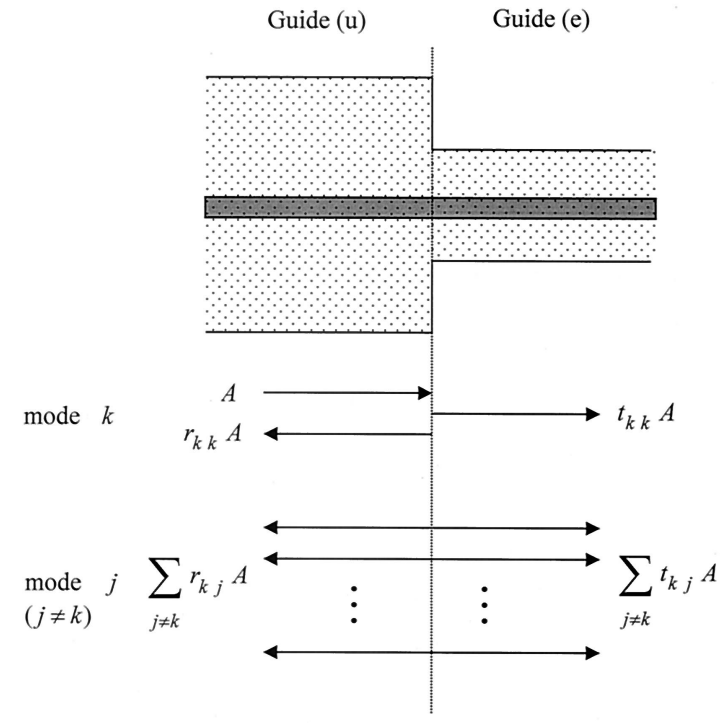

(a)

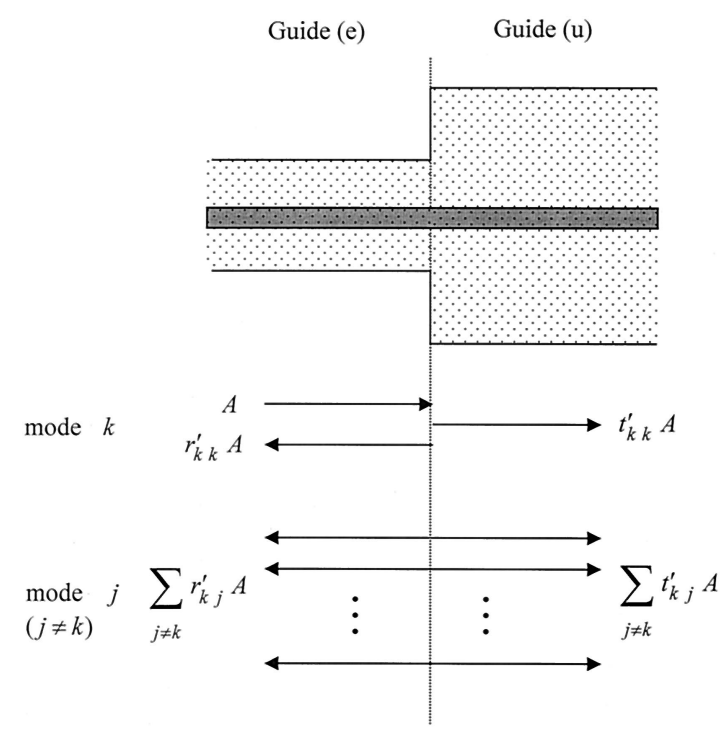

(b)

Fig. 3. (a) Schematic diagram of scattering of mode $k$ as a result of the heterojunction from the unetched region to the etched region. Also shown is the definition of the corresponding scattering coefficients. (b) Same as (a), except for mode incidence from the etched region to the unetched region.

Before we introduce the self-consistent approximation, for simplicity we define the following overlapping integrals:

$$
\begin{aligned}
I_{k j} & =(1 / 2) \int_{A_{\infty}}\left[\mathbf{e}_{k}^{(u)} \times \mathbf{h}_{j}^{(e) *}\right] \cdot \mathbf{z d} A, \\
J_{k j} & =(1 / 2) \int_{A_{\infty}}\left[\mathbf{e}_{j}^{(e) *} \times \mathbf{h}_{k}^{(u)}\right] \cdot \mathbf{z d} A .
\end{aligned}
$$

These overlapping integrals can be transformed into clearer forms by use of the reciprocity theorem discussed in Appendix A. Here we let the unprimed 
notation represent the waveguide modes of the unetched region and the primed notation belong to the etched region. Using Eqs. (A10) and (A12) and the orthogonality conditions for modes of etched regions, we have

$$
\begin{gathered}
I_{k j}=a_{k j}+b_{k j}, \\
J_{k j}=a_{k j}-b_{k j},
\end{gathered}
$$

with the following expressions for $a_{k j}$ and $b_{k j}$ :

$$
\begin{aligned}
a_{k j} & =\frac{k_{0}}{4\left[\beta_{k}^{(u)}-\beta_{j}^{(e)}\right]} \frac{1}{Z_{0}} \int_{A_{\infty}}\left\{\left[n_{t}^{(u)}\right]^{2}-\left[n_{t}^{(e)}\right]^{2}\right\} \mathbf{e}_{t k}^{(u)} \cdot \mathbf{e}_{t j}^{(e) *} \mathrm{~d} A, \\
b_{k j} & =\frac{k_{0}}{4\left[\beta_{k}^{(u)}+\beta_{j}^{(e)}\right]} \frac{1}{Z_{0}} \int_{A_{\infty}}\left\{\left[n_{t}^{(u)}\right]^{2}-\left[n_{t}^{(e)}\right]^{2}\right\} \mathbf{e}_{t k}^{(u)} \cdot \mathbf{e}_{t j}^{(e) *} \mathrm{~d} A .
\end{aligned}
$$

The contributions from the longitudinal component are again neglected here. We now rewrite continuity equations (12) and (13) as follows:

$$
\begin{aligned}
& \left(1+r_{k k}\right) \mathbf{e}_{t k}^{(u)}=t_{k k} \mathbf{e}_{t k}^{(e)}+\sum_{k \neq j}\left(t_{k j}-r_{k j}\right) \mathbf{e}_{t j}^{(e)}+\delta \mathbf{e}_{k}, \\
& \left(1-r_{k k}\right) \mathbf{h}_{t k}^{(u)}=t_{k k} \mathbf{h}_{t k}^{(e)}+\sum_{j \neq k}\left(t_{k j}+r_{k j}\right) \mathbf{h}_{t j}^{(e)}+\delta \mathbf{h}_{k},
\end{aligned}
$$

where

$$
\begin{aligned}
\delta \mathbf{e}_{k} & =\sum_{j \neq k} r_{k j}\left[\mathbf{e}_{t j}^{(e)}-\mathbf{e}_{t j}^{(u)}\right], \\
\delta \mathbf{h}_{k} & =\sum_{j \neq k} r_{k j}\left[\mathbf{h}_{t j}^{(u)}-\mathbf{h}_{t j}^{(e)}\right] .
\end{aligned}
$$

In what follows, we neglect these two parts of the transverse fields. This approximation is plausible either because of the small reflection coefficients or as a result of the similarity of mode fields of the two waveguides.

Using the orthogonality conditions for mode fields of the etched region, we obtain the following results: First, for mode $k$ we have

$$
t_{k k}=I_{k k}\left(1+r_{k k}\right)=J_{k k}\left(1-r_{k k}\right) .
$$

We can use this identity to solve the self-reflection coefficient $r_{k k}$ :

$$
r_{k k}=\frac{J_{k k}-I_{k k}}{J_{k k}+I_{k k}}=-\frac{b_{k k}}{a_{k k}},
$$

where Eqs. (15) have been used for the last identity. If the contributions of the $z$ component to coefficients $a_{k k}$ and $b_{k k}$ can be neglected, as in Eqs. (16), the self-reflection coefficient can further be simplified to

$$
r_{k k}=\frac{\beta_{k}^{(e)}-\beta_{k}^{(u)}}{\beta_{k}^{(e)}+\beta_{k}^{(u)}},
$$

which is similar to the familiar Fresnel formula for normal incidence on a heterointerface of homogeneous media.

As is shown in Section 5 below, the propagation constants of the fundamental core mode and the first few cladding modes in the unetched and etched regions are similar. Thus the self-reflection coefficients are small, confirming our initial approximation. Then we apply the orthogonality conditions for mode $j$ in Eqs. (17) and (18) to get

$$
\begin{aligned}
\left(1+r_{k k}\right) I_{k j} & =t_{k j}-r_{k j}, \\
\left(1-r_{k k}\right) J_{k j} & =t_{k j}+r_{k j} .
\end{aligned}
$$

The cross-scattering coefficients can thus be solved as

$$
\begin{aligned}
t_{k j} & =(1 / 2)\left[\left(J_{k j}+I_{k j}\right)-r_{k k}\left(J_{k j}-I_{k j}\right)\right]=a_{k j}+r_{k k} b_{k j}, \\
r_{k j} & =(1 / 2)\left[\left(J_{k j}-I_{k j}\right)-r_{k k}\left(J_{k j}+I_{k j}\right)\right] \\
& =-b_{k j}-r_{k k} a_{k j} .
\end{aligned}
$$

If the propagation constants of the mutually coupled modes are similar, i.e., $\beta_{j} \cong \beta_{k}$, the coefficients $b_{k j} \ll$ $a_{k j}$, and thus the reflection coefficients $r_{k j}$, are much smaller than the transmission coefficients. This result confirms that our approach is self-consistent. We can use similar approaches to derive the scattering coefficients for incidence from the etched to the unetched region, i.e., $t_{k k}^{\prime}, r_{k k}^{\prime}, t_{k j}^{\prime}$, and $r_{k j}^{\prime}$.

\section{Forward-Transfer Matrices for a Corrugated Long-Period Fiber Grating}

The transfer-matrix method is a powerful tool for describing the propagation of wave fields in a binarylike periodic structure. For example, based on local-mode matching, one can use a set of transfer matrices to model grating-assisted codirectional couplers. ${ }^{17}$ Recently we also utilized the transfermatrix method and perturbation expansions to model a photoinduced binary LPFG. ${ }^{10}$ In these two applications of the transfer-matrix method, backward reflection is assumed to be negligible, and in Ref. 10 it was shown that such is indeed the case for photoinduced long-period gratings. As we discussed in Section 3 , the reflection coefficients $r_{k k}$ and $r_{k j}$ are much smaller than the corresponding transmission coefficients $t_{k k}$ and $t_{k j}$. Thus we may neglect the reflected amplitudes and use a set of forward-transfer matrices to describe the evolution of mode amplitudes through the corrugated LPFG.

For the corrugated structure shown in Fig. 1, two coupling mechanisms are in effect. First, the corrugated cladding structure introduces periodic waveguide discontinuities along the fiber, and couplings between modes occur as a result of these discontinuities. Second, when the corrugated LPFG is under external tensile stress, a periodic strain distribution is induced within the corrugated structure, as discussed in Section 2. And, because of the photoelastic effect, this differential strain will further introduce a periodic index modulation along the fiber, which also contributes to mode coupling. However, the effect of the first mechanism is relatively negligible compared with that of the second. The reason for this is as follows: The coupled modes of our main concern are the fundamental core mode and the 
phase-matched cladding modes, which are of the first few mode orders. The fields of these modes are distributed mainly within regions near the fiber core. Thus, changes in the outer cladding radius have little effect on these centrally confined modes. And this is also shown by our experimental result that the transmission loss is almost negligible when the corrugated LPFG is free from any tensile strain. Mathematically, we may assume that these modes, although they belong to different waveguide structures, are quasi-orthogonal, as we discuss below.

The dependence of the transmission coefficients on the applied tensile strain is now investigated as follows: We introduce another important assumption, i.e., that the mode fields of the two regions are quasiorthogonal. This is so because, for the first few confined modes, the fields of the same mode order are quite similar; e.g., there are the similar mode profiles, nearby node points, etc. Thus the overlapping integrals $\bar{I}_{k j}$ and $\bar{J}_{k j}$ of the unperturbed mode fields of the two regions have the following properties: The overlaps of different modes are relatively smaller than the self-overlaps; e.g., $\bar{I}_{k j} \ll \bar{I}_{k k}, \bar{I}_{j j}$. And clearly this holds only for those modes that are confined near the core region. For higher-order cladding modes, this assumption will not be valid. However, because the corrugated LPFG is designed mainly to permit resonance coupling between the core mode and the first few centrally confined cladding modes, we may assume that this property is valid as far as those modes are concerned.

In Section 5 we shall show numerically that such is indeed the case for the dispersion-shifted fiber used in our experiment. Thus, for simplicity, we may express this property mathematically as

$$
\bar{I}_{k j} \cong \bar{J}_{k j} \cong \delta_{k j} \tau_{k}
$$

where $\tau_{k}$ is defined as the overlapping integral of the unperturbed fields of the two basic regions for mode $k$. Substituting expressions for the perturbed mode fields [expressions (8)] into Eqs. (20) and (24) for transmission coefficients and using quasi-orthogonality assumption (25), we have

$$
t_{k j} \cong \delta_{k j}\left(1-\alpha_{k} s_{z}^{2}\right) \tau_{k}+s_{z} \sigma_{k j} \text {. }
$$

Here we keep terms up to the second order in strain $s_{z}$ in the diagonal term for consistency and define the following parameters:

$$
\begin{aligned}
& \alpha_{k}=d_{k}^{(u)}+d_{k^{\prime}}^{(e)}\left[\frac{a_{\mathrm{cl}}^{(u)}}{a_{\mathrm{cl}}^{(e)}}\right]^{4}, \\
& \sigma_{k j}=\left\{\begin{array}{cc}
{\left[\frac{a_{\mathrm{cl}}^{(u)}}{a_{\mathrm{cl}}^{(e)}}\right]^{2} \frac{\kappa_{k j}^{(e)} \tau_{k}}{\left[\bar{\beta}_{k}^{(e)}-\bar{\beta}_{j}^{(e)}\right]}-\frac{\kappa_{k j}^{(u)} \tau_{j}}{\left[\bar{\beta}_{k}^{(u)}-\bar{\beta}_{j}^{(e)}\right]}} & (k \neq j) \\
0 & (k=j)
\end{array} .\right.
\end{aligned}
$$

In deriving Eq. (28) we used $\kappa_{j k}=\kappa_{k j}$. We also define $\sigma_{j k}{ }^{\prime} \equiv-\sigma_{j k}$, which will be used below and has the property that $\sigma_{k j} \sigma_{j k}{ }^{\prime}>0$ for any pair $j$ and $k$. Inasmuch as the mode fields involved are similar, as discussed above, we have $\kappa_{k j}^{(u)} \cong \kappa_{k j}^{(e)}$, and the self-

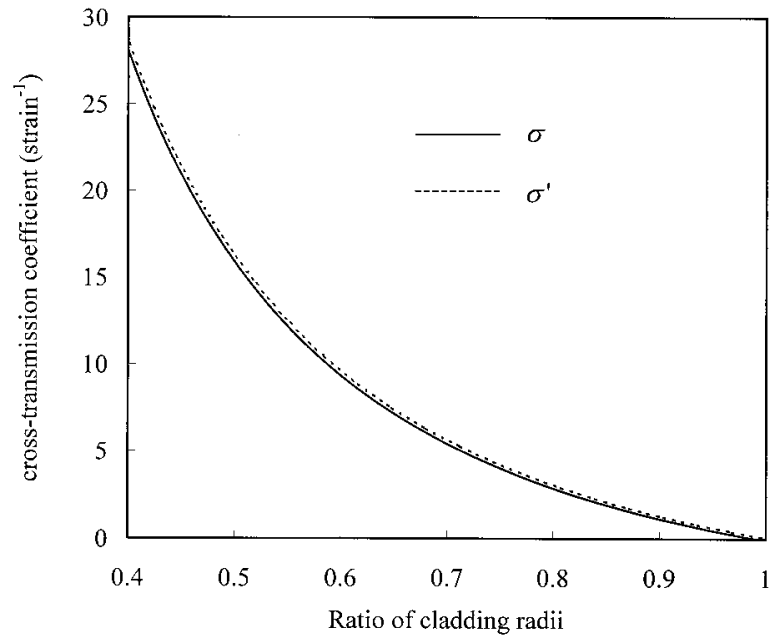

Fig. 4. Dependence of cross-transmission coefficients $\sigma \equiv \sigma_{\text {co-cl }}$ and $\sigma^{\prime} \equiv \sigma_{\mathrm{cl}-\mathrm{co}}{ }^{\prime}$ on the ratio of the cladding radii $a_{\mathrm{cl}}{ }^{(e)} / a_{\mathrm{cl}}{ }^{(u)}$.

transmission coefficients are all close to unity; otherwise the loss would be appreciable. Thus we see that, when $a_{\mathrm{cl}}^{(u)} \cong a_{\mathrm{cl}}^{(e)}$, the parameters $\sigma_{k j}$ will be small, and the cross couplings will be negligible, as confirmed from the experiment. In Fig. 4 we show the dependence of cross-transmission coefficients $\sigma_{k j}$ on the ratio of the cladding radii, $a_{\mathrm{cl}}^{(e)} / a_{\mathrm{cl}}^{(u)}$. In the figure we have defined $\sigma \equiv \sigma_{\text {co-cl }}$ and $\sigma^{\prime} \equiv \sigma_{\text {cl-co }}$ for the fundamental core mode and the first cladding mode. The fiber parameters are described in Section 5, and we take $a_{\mathrm{cl}}^{(u)}=62.5 \mu \mathrm{m}$ here. Note that the cross-transmission coefficients grow superlinearly as the ratio decreases below 1 , where these coefficients vanish. Therefore, for efficient cross coupling, the cladding radius contrast must be large. From relation (26) we can see that when there is no applied tensile stress, $s_{z}=0$; under the assumption of quasi-orthogonality, the cross transmission is almost zero, which is consistent with the experimental observation. As the applied strain increases, the cross couplings also grow linearly, where the proportional constant is $\sigma_{k j}$. Besides, self-transmission also decreases as a result of coupling to other modes, which is of second order in $s_{z}$.

We now apply the above results to the case of almost resonant coupling between the core mode and a phase-matched cladding mode. As a result of the phase-matching condition, the power is distributed mainly between these two modes, and we may neglect couplings to the other modes. If the resonance wavelengths are sufficiently separated from one another, the effect of the other modes can be regarded as a dissipative loss to these two nearly phase-matched modes.

Let the mode amplitudes in region $r$ be represented by a column vector, $\mathbf{A}^{(r)}=\left[A_{\mathrm{co}}^{(r)}, A_{\mathrm{cl}}^{(r)}\right]^{T}$, where $r=u$ and $r=e$ for unetched and etched regions, respectively. Then the propagation of the wave field can be described by the following transfer matrices: For crossing the interface from the unetched region to the etched region, the mode amplitudes are connected by 
the matrix equation $\mathbf{A}^{(e)}=\mathbf{F}^{(e \mid u)}\left(s_{z}\right) \cdot \mathbf{A}^{(u)}$, where $2 \times$ 2 matrix $\mathbf{F}^{(e \mid u)}$ is given by

$$
\mathbf{F}^{(e \mid u)}\left(s_{z}\right)=\left[\begin{array}{cc}
\left(1-\alpha_{\mathrm{co}} s_{z}{ }^{2} \tau_{\mathrm{co}}\right) & \sigma s_{z} \\
-\sigma^{\prime} s_{z} & \left(1-\alpha_{\mathrm{cl}} s_{z}{ }^{2}\right) \tau_{\mathrm{cl}}
\end{array}\right],
$$

where $\sigma$ and $\sigma^{\prime}$ are the corresponding cross-coupling coefficients between these two modes as defined in Eq. (28) and Fig. 4. Similarly, for incidence from the etched region to the unetched region the mode amplitude changes according to $\mathbf{A}^{(u)}=\mathbf{F}^{(u \mid e)}\left(s_{z}\right) \cdot \mathbf{A}^{(e)}$ with the following interface matrix:

$$
\mathbf{F}^{(u \mid e)}\left(s_{z}\right)=\left[\begin{array}{cc}
\left(1-\alpha_{\mathrm{co}} s_{z}{ }^{2}\right) \tau_{\mathrm{co}} & -\sigma^{\prime} s_{z} \\
\sigma s_{z} & \left(1-\alpha_{\mathrm{cl}} s_{z}^{2}\right) \tau_{\mathrm{cl}}
\end{array}\right] .
$$

Within the two regions there is no coupling between the modes, and the amplitudes acquire only phase changes. This can be described by use of the phase matrices as $\mathbf{A}^{(r)}\left[\Lambda^{(r)}\right]=\mathbf{P}^{(r)} \cdot \mathbf{A}^{(r)}(0)$ for region $r$, and the phase matrices are given by $(r=u$ or $r=e)$

$$
\mathbf{P}^{(r)}=\left[\begin{array}{cc}
\exp \left[i \theta_{\mathrm{co}}^{(r)}\right] & 0 \\
0 & \exp \left[i \theta_{\mathrm{cl}}^{(r)}\right]
\end{array}\right],
$$

where the phase shifts are

$$
\begin{aligned}
& \theta_{\mathrm{co}}^{(r)}=\left[\overline{\boldsymbol{\beta}}_{\mathrm{co}}^{(r)}-s_{z}^{(r)} \kappa_{\mathrm{co}-\mathrm{co}}^{(r)}\right] \Lambda^{(r)}\left[1+s_{z}^{(r)}\right], \\
& \theta_{\mathrm{cl}}^{(r)}=\left[\bar{\beta}_{\mathrm{cl}}^{(r)}-s_{z}^{(r)} \kappa_{\mathrm{cl}-\mathrm{cl}}^{(r)}\right] \Lambda^{(r)}\left[1+s_{z}^{(r)}\right] .
\end{aligned}
$$

The couplings through the propagation of a unit period can thus be described by use of the cascaded matrix $\mathbf{F}\left(s_{z}\right)=\mathbf{P}^{(u)} \cdot \mathbf{F}^{(u \mid e)} \cdot \mathbf{P}^{(e)} \cdot \mathbf{F}^{(e \mid u)}$. As discussed in Ref. 10, the phase-matching condition can be expressed as follows:

$$
\left[\theta_{\mathrm{co}}^{(u)}-\theta_{\mathrm{cl}}^{(u)}\right]+\left[\theta_{\mathrm{co}}^{(e)}-\theta_{\mathrm{cl}}^{(e)}\right]=2 \pi .
$$

As can be seen from the following numerical example, the quantities of the unetched region are fairly close to those of the etched region for core and cladding modes of the few first orders. For such an approximation and when $\Lambda^{(u)}=\Lambda^{(e)}=\Lambda / 2$, phasematching condition (33) can be written as

$$
\bar{\beta}_{\mathrm{co}}-\bar{\beta}_{\mathrm{cl}}-\bar{s}_{z}\left(\kappa_{\mathrm{co}-\mathrm{co}}-\kappa_{\mathrm{cl}-\mathrm{cl}}\right)=\left(1-\bar{s}_{z}\right) \frac{2 \pi}{\Lambda},
$$

where $\bar{\varepsilon}=\left(\varepsilon+\varepsilon^{\prime}\right) / 2$ is the averaged strain of the corrugated structure. The resonance wavelength can be approximated as

$$
\lambda_{0}=\Lambda\left(\bar{n}_{\mathrm{co}}-\bar{n}_{\mathrm{cl}}\right)\left[1+\bar{s}_{z}\left(1-\frac{\Delta \kappa_{s} \Lambda}{2 \pi}\right)\right],
$$

where $\bar{n}_{\mathrm{co}}$ and $\bar{n}_{\mathrm{cl}}$ are the effective indices of the unperturbed core and the averaged cladding modes, respectively, and $\Delta \kappa_{s}=\kappa_{\mathrm{co}-\mathrm{co}}-\kappa_{\mathrm{cl}-\mathrm{cl}}$ is the difference of the self-coupling coefficients. As is shown below, usually $\Delta \kappa_{s}>0$. From Eq. (35), the applied strain has two effects on the resonance wavelength. One is the geometric elongation of the corrugated period $\bar{\varepsilon} \Lambda$, and the other is the contribution from strain-induced

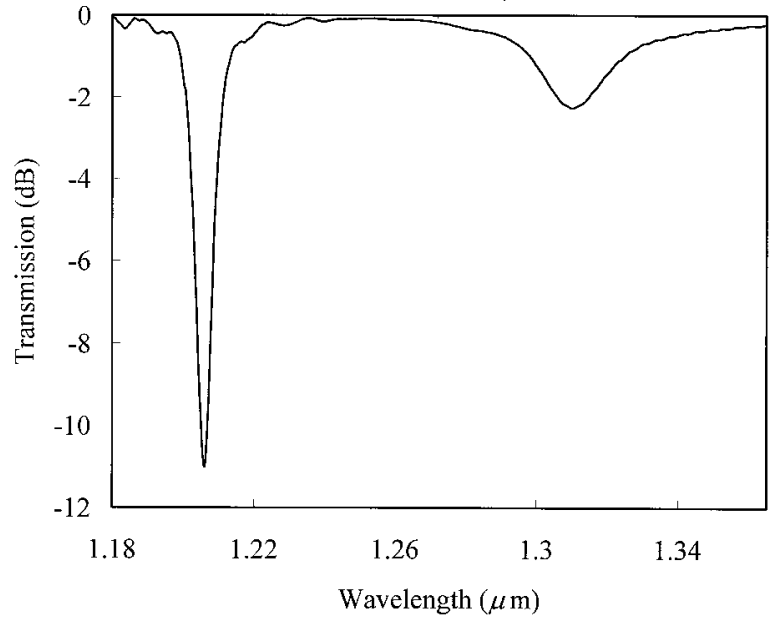

Fig. 5. Transmission spectrum of a corrugated LPFG under applied strain $s_{z}=530 \mu$ strain.

self-couplings of mode fields. These effects are discussed in Section 5.

\section{Simulation Results and Comparison}

In this section we apply the formulation of Section 4 to the analysis of a specific corrugated LPFG and compare the results with the measured spectra. Figure 2 is a schematic diagram of the experimental setup for measuring the transmission spectrum of a corrugated LPFG. A dispersion-shifted fiber of length $L$ is fixed at one end, and the other end is stretched by applied tensile force $F$ to make a $\Delta L$ elongation. A corrugated section with length $N \Lambda$ lies in the middle of the fiber. As discussed above, when $N \Lambda \ll L$, the longitudinal strain of the unetched regions is equal to the measured strain; i.e. $\varepsilon$ $\cong \Delta L / L$. The period of the corrugated structure is $\Lambda=350 \mu \mathrm{m}$. And for simplicity the lengths of etched and unetched regions are chosen to be equal, $\Lambda^{(u)}=\Lambda^{(e)}=\Lambda / 2$. The etching process is described in Ref. 8 , and the etched cladding radius is $a_{\mathrm{cl}}^{(e)} \cong 27$ $\mu \mathrm{m}$, whereas the original unetched radius is $a_{\mathrm{cl}}^{(u)}=$ $62.5 \mu \mathrm{m}$.

Figure 5 shows the measured transmission spectrum of the corrugated LPFG with applied strain $\varepsilon=$ $530 \mu$ strain. There are two resonant loss dips in the wavelength range shown in Fig. 5; one is at $1.206 \mu \mathrm{m}$ and the other is at $1.313 \mu \mathrm{m}$. The two dips are attributed to couplings from the fundamental core mode to two different cladding modes. The fiber used in our experiments has a normalized index difference $\Delta \cong 0.0077$ and thus can be regarded as a weakly guiding structure. The fundamental core mode is designated $\mathrm{LP}_{01}^{(\mathrm{co})}$, which is the linearly polarized approximation of the exact $\mathrm{HE}_{11}$ mode. From the above discussion, the off-diagonal transmission coefficients $\sigma$ and $\sigma^{\prime}$ are proportional to the cross-coupling constants $\kappa_{\mathrm{co}-\mathrm{cl}}^{(u)}$ and $\kappa_{\mathrm{co}-\mathrm{cl}}^{(e)}$. As the strain-induced index perturbation is uniform over the cross section of the etched or unetched region, the coupled cladding modes should have the same axial 


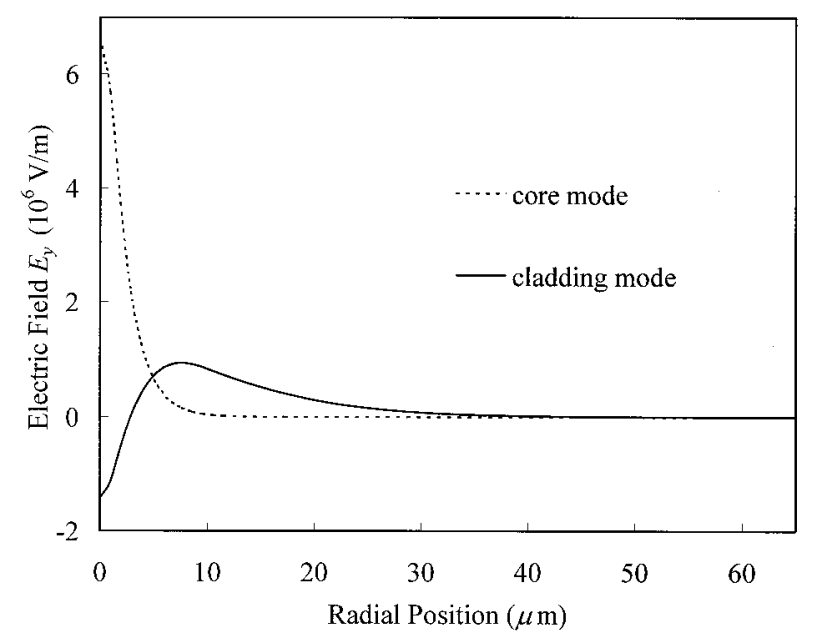

Fig. 6. Electric fields of the core mode and the first cladding mode in the unetched region.

symmetry as the fundamental core mode; otherwise the integrals for $\kappa_{\mathrm{co-cl}}[\mathrm{Eq}$. (9)] would vanish. Thus the cladding modes can be designated in the linear polarized approximation as $\mathrm{LP}_{0 v}^{(c l)}$, corresponding to the exact $\mathrm{HE}_{1 v}$ modes for $v=2,3, \ldots$.

Because the resonance wavelength ranges of the two cladding modes are sufficiently separated, we consider mainly coupling to the first cladding mode, i.e., $\mathrm{LP}_{01}^{(\mathrm{co})} \leftrightarrow \mathrm{LP}_{02}^{(\mathrm{cl})}$ in the following discussion. The index profile of the dispersion-shifted fiber used in our calculation for best fit has a core region that can be approximated by a Gaussian envelope with peak core index 1.473 and a radius of $\cong 2.45 \mu \mathrm{m}$, an exponential-tailed transition region of width $\cong 7 \mu \mathrm{m}$, followed by a cladding region with index 1.4617 . Figure 6 shows the calculated electric field $E_{y}$ for the core mode $\left(\mathrm{LP}_{01}\right)$ and the first cladding mode $\left(\mathrm{LP}_{02}\right)$ of the unetched region. The calculation is based on the basic finite-difference method for solving waveguide modes. ${ }^{18}$ The core mode is confined principally in the core and has a full width at half-maximum of approximately $5 \mu \mathrm{m}$. From our calculation, the core mode is almost independent of cladding radius $a_{\mathrm{cl}}$ in that the etched radius is not too close to the core region. In our case the etched region has a cladding radius $a_{\mathrm{cl}}^{(e)} \cong 27 \mu \mathrm{m}$, which has little effect on the core mode. Thus the core modes of the two regions are taken to be the same in what follows.

The first cladding mode has a node at $r=2.7 \mu \mathrm{m}$, and the amplitude starts to decay after the turning point at approximately $r=8 \mu \mathrm{m}$. The mode field is shown to be more closely confined to the core than that of the step-index fiber. ${ }^{9}$ In Fig. 7 we show the electric fields $E_{y}$ of the cladding modes in the unetched and the etched regions. It can be seen that, as the cladding radius decreases, the mode field of the first cladding mode is pushed inward and the amplitude almost vanishes for $r>a_{\mathrm{cl}}$. However, the overlap of these two fields is still large. Because the mode fields are still quite well matched, as discussed in Section 4, we may neglect reflections

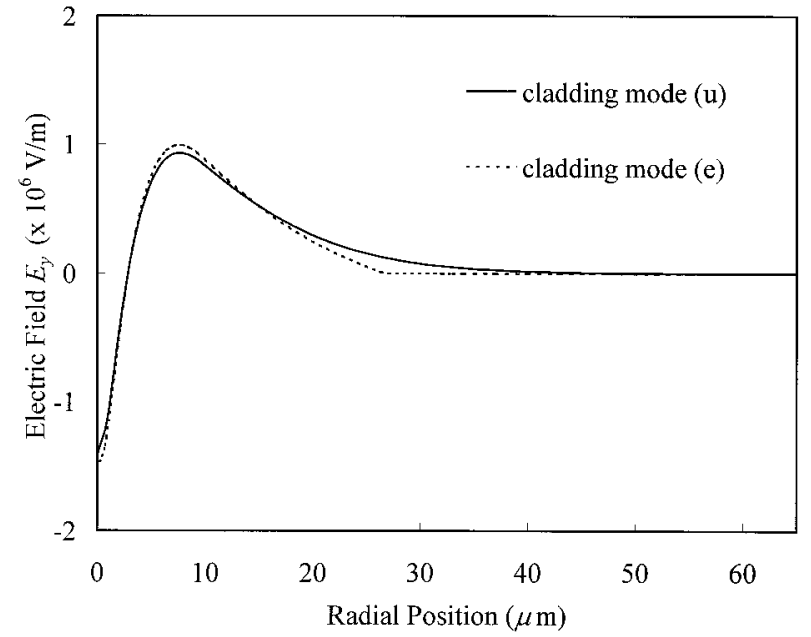

Fig. 7. Comparison of the electric fields of cladding modes in unetched and etched regions.

at the heterointerfaces of the unetched and etched regions.

The dispersion curves of effective indices of the core mode and the cladding modes (for both etched and unetched regions) are shown in Fig. 8. It is found that the effective indices of the cladding modes are fairly close for etched and unetched regions.

Figure 9 shows the dispersion curves of overlapping integrals $\tau_{\mathrm{co}}$ and $\tau_{\mathrm{cl}}$ defined in relation (25) for the fundamental core mode and the first cladding mode. The overlap of core modes is almost unity, whereas the overlap of cladding modes decreases for larger wavelengths. The nonunity feature of the cladding-mode overlap accounts for losses of coupling to higher-order modes and radiation modes as a result of waveguide discontinuity, which cannot be taken into consideration in coupled-mode theory.

Figure 10 shows the dispersion of self-coupling constants $\kappa_{\mathrm{co}-\mathrm{co}}, \kappa_{\mathrm{cl}-\mathrm{cl}}^{(u)}$, and $\kappa_{\mathrm{cl}-\mathrm{cl}}^{(e)}$ defined in Eqs. (9) and (11). As can be seen, the self-coupling constants are

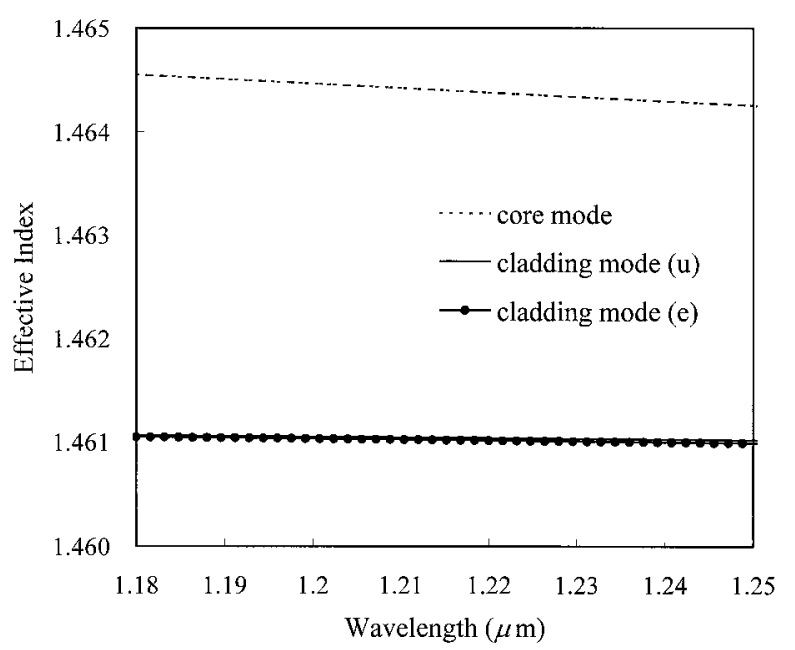

Fig. 8. Dispersion curves of effective indices for core and cladding modes in unetched and etched regions. 


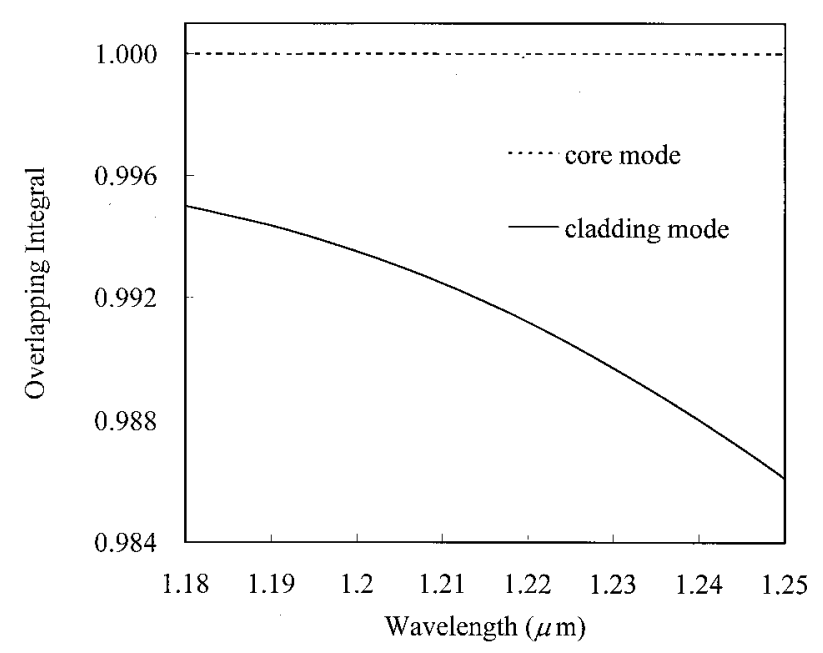

Fig. 9. Dispersions of overlapping integrals $\tau_{\text {co }}$ for the core mode and $\tau_{\mathrm{cl}}$ for the cladding mode.

almost the same for cladding modes of the unetched and the etched regions; i.e., $\kappa_{\mathrm{cl}-\mathrm{cl}}^{(u)} \cong \kappa_{\mathrm{cl}-\mathrm{cl}}^{(e)}$. And the self-coupling constant of the core mode is larger than that of cladding modes. These coupling coefficients will influence the phase-matching condition, as shown in Eqs. (34) and (35). The difference $\Delta \kappa_{s}$ is found from Fig. 10 to be approximately $0.019 \mu \mathrm{m}^{-1}$ for photoelastic constant $p_{e}=0.193$. For $\Lambda=350$ $\mu \mathrm{m}$, the contribution of self-coupling to the wavelength shift is $\Delta \kappa_{s} \Lambda / 2 \pi \approx 1$. Thus, from Eq. (35), the overall strain-induced wavelength shift is quite small.

The dispersion curves of the cross-coupling constants, $\kappa_{\mathrm{co}-\mathrm{cl}}^{(u)}$ and $\kappa_{\mathrm{co}-\mathrm{cl}}^{(e)}$, for unetched and etched regions are shown in Fig. 11. Both curves decrease with increasing wavelength. The cross-coupling coefficient of the etched region is larger than that of the unetched region because the cladding-mode field is

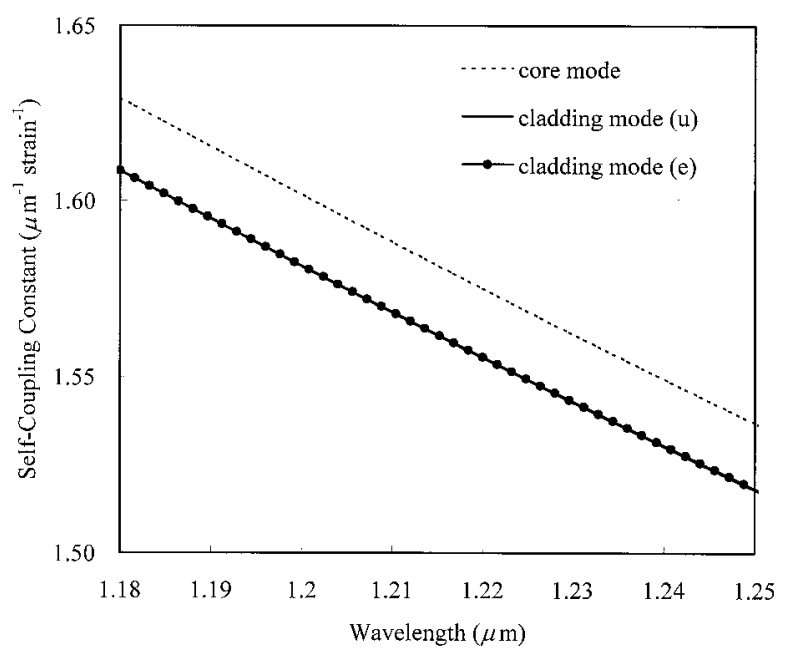

Fig. 10. Dispersions of self-coupling constants $\kappa_{\mathrm{co}-\mathrm{co}}$ for the core mode and $\kappa_{\mathrm{cl}-\mathrm{cl}}^{(u)}$ and $\kappa_{\mathrm{cl}-\mathrm{cl}}^{(e)}$ for the cladding modes in unetched and etched regions.

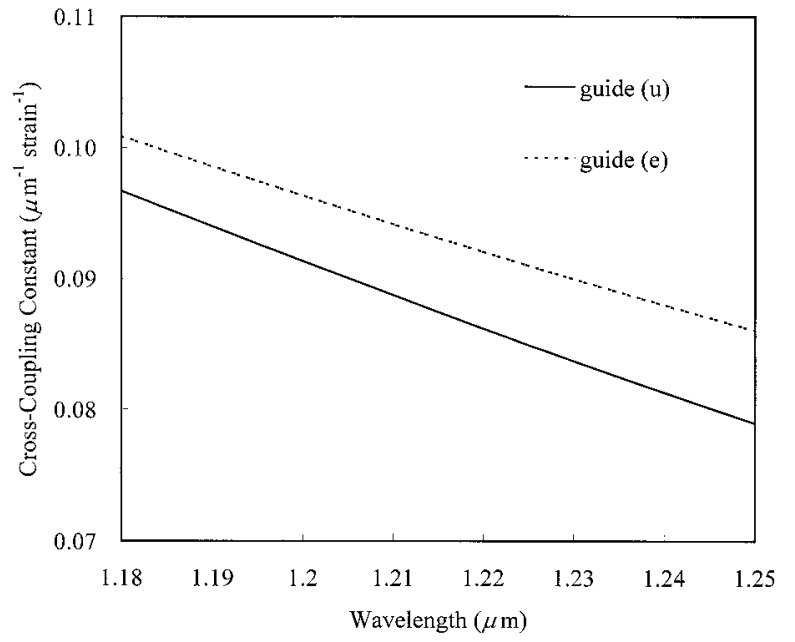

Fig. 11. Dispersions of cross-coupling constants $\kappa_{\mathrm{co}-\mathrm{cl}}{ }^{(u)}$ and $\kappa_{\mathrm{co}-}$ $\mathrm{cl}^{(e)}$ in unetched $(\mathrm{u})$ and etched (e) regions.

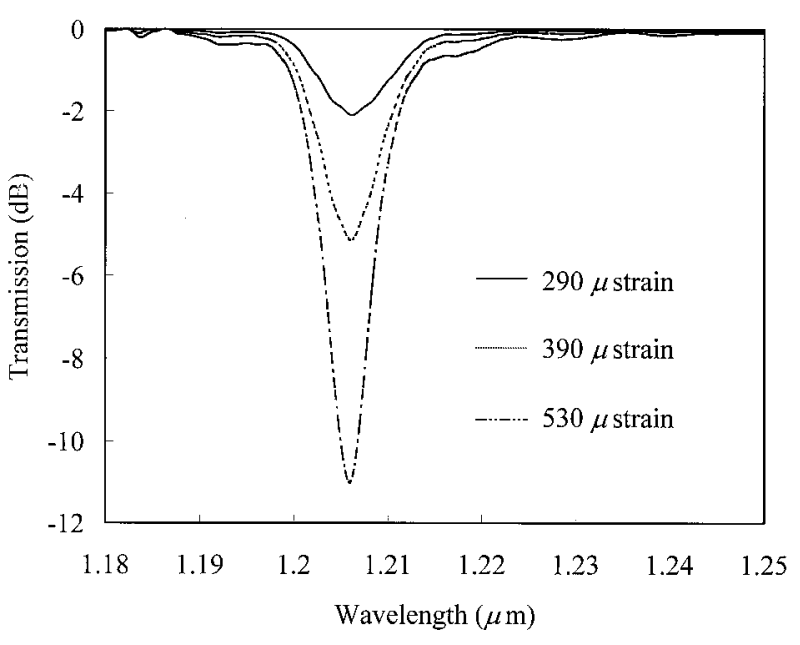

(a)

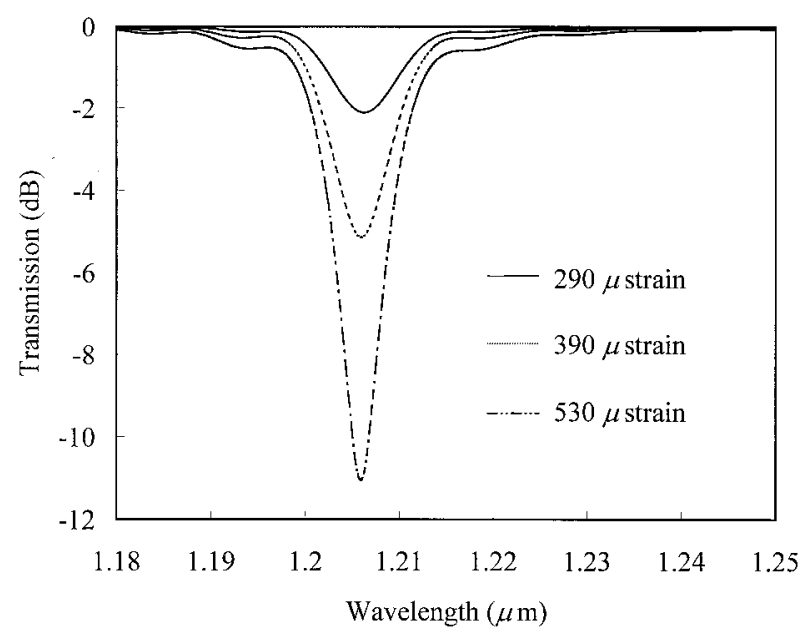

(b)

Fig. 12. (a) Measured transmission spectra of the corrugated LPFG under various applied strains. (b) The corresponding calculated transmission spectra. 
more closely confined to the core region as the cladding radius decreases, as confirmed from Fig. 7.

Figure 12(a) shows the measured transmission spectrum of the corrugated LPFG for coupling to the first cladding mode under applied strains $s_{z}=290$, 390, $530 \mu$ strain. The transmission spectra have two notable features. First, the transmission loss increases as the applied strain, and this makes the corrugated LPFG a tunable loss filter. Second, the resonance dip wavelength is almost independent of the applied tensile strain. These properties are different from those of the conventional photoinduced LPFG, in which the resonance wavelength shifts to longer wavelengths as the index modulation is increased. ${ }^{19}$ In Fig. 12(b) we show the calculated transmission spectra that result from the transfermatrix approach described in Sections 2-4. As can be seen, the calculated spectra agree quite well with the measured ones. The spectrum has no complete node, as would be predicted from coupled-mode theory, and this is attributed to the nonunity of the cladding-mode overlapping integral. The independence of resonance wavelength on strain is explained above: Because the self-coupling coefficients are similar for core and cladding modes, the straininduced corrections to the propagation constants of the core mode are compensated for by those of the cladding mode and by the geometrical elongation of the grating period.

\section{Conclusion}

A transfer-matrix method based on the modematching technique has been applied to modeling of a corrugated LPFG. The operation of the corrugated LPFG is based on the photoelastic effect and on the strain difference between the unetched and the etched regions when a tensile force is applied to the corrugated structure. Because of the inverse proportionality of the strain to the cross-sectional areas, a periodic strain distribution, and equivalently an index modulation, is induced in the corrugated structure. As the etched cladding radius is much smaller than the unetched radius, the effect of the corrugated structure on cladding modes cannot be treated as a small perturbation. We have presented a modal analysis to describe the coupling of mode amplitudes through the heterointerfaces of unetched and etched regions. The mode fields are solved by the finitedifference method for the unetched and the etched regions independently, and the mode fields obtained are regarded as unperturbed fields with respect to the strain-induced index perturbation. The perturbed mode fields of these two basic regions are derived from the unperturbed fields by use of the first-order (linear in strain) vectorical perturbation expansion. By neglecting backreflection, we have presented a forward transfer-matrix method to model the mode couplings within the corrugated structure. Two interface matrices were introduced for mode couplings. The off-diagonal elements of these interface matrices account for the strain-induced coupling between the core and cladding modes and are found to be linearly proportional to strain. Propagation through the unetched and the etched regions is described by two phase matrices, which have only diagonal elements to account for the propagation phase difference. The calculated spectra based on the transfer-matrix method have been compared with the experimental results and are in fairly good agreement. The transmission loss grows with increasing strain, whereas the resonance peak wavelength is almost fixed. All these features are explained well by our model.

\section{Appendix A. Reciprocity Theorem for Uniaxially Anisotropic Waveguides}

The reciprocity theorem is used to connect two distinct waveguide problems and can serve as a basis for perturbation expansions of the mode fields. A detailed discussion of the reciprocity theorem for isotropic waveguides can be found in Ref. 13. Inasmuch as the index tensor of waveguides under tensile strain will be slightly uniaxial anisotropic, here we give reciprocity formulas for uniaxial waveguides with the optical axis parallel to the guiding $z$ axis. For an anisotropic medium the displacement vector is related to the electric field as

$$
\mathbf{D}=\varepsilon_{0} \mathbf{n}^{2} \cdot \mathbf{E},
$$

where $\mathbf{n}^{2}$ is a tensor with nine components and for a uniaxial waveguide has the following form:

$$
\mathbf{n}^{2}=\left[\begin{array}{ccc}
n_{t}{ }^{2} & 0 & 0 \\
0 & n_{t}{ }^{2} & 0 \\
0 & 0 & n_{z}{ }^{2}
\end{array}\right] .
$$

Consider two waveguide electromagnetic fields characterized by index tensors $\mathbf{n}^{2}$ and $\mathbf{n}^{\prime 2}$. In what follows, we use index tensor $\mathbf{n}^{2}$ to denote the guiding structure of mode fields without a prime and $\mathbf{n}^{\prime 2}$ to denote that of primed fields. The electric and magnetic fields the correspond to these two situations are

$$
\begin{aligned}
\mathbf{E} & =\mathbf{e}_{j} \exp \left(i \beta_{j} z\right), \\
\mathbf{H} & =\mathbf{h}_{j} \exp \left(i \beta_{j} z\right),
\end{aligned}
$$

which is mode $j$ of the $\mathbf{n}^{2}$ waveguide, and

$$
\begin{aligned}
\mathbf{E}^{\prime} & =\mathbf{e}_{k}{ }^{\prime} \exp \left(i \beta_{k}{ }^{\prime} z\right), \\
\mathbf{H}^{\prime} & =\mathbf{h}_{k}{ }^{\prime} \exp \left(i \beta_{k}{ }^{\prime} z\right),
\end{aligned}
$$

which is mode $k$ of the $\mathbf{n}^{\prime 2}$ waveguide. These mode fields are assumed to be orthogonal and normalized to carry unity power. We define a vector function $\mathbf{F}_{c}$ by

$$
\mathbf{F}_{c}=\mathbf{E} \times \mathbf{H}^{\prime *}+\mathbf{E}^{\prime *} \times \mathbf{H} .
$$

The conjugated form of the reciprocal theorem is given by

$$
\frac{\partial}{\partial z} \int_{A_{\infty}} \mathbf{F}_{c} \cdot \mathbf{z d} A=\int_{A_{\infty}} \nabla \cdot \mathbf{F}_{c} \mathrm{~d} A .
$$


Substituting Eqs. (A3)-(A5) into the above identity, and using the following Maxwell equations for $\mathbf{E}$ and H:

$$
\begin{aligned}
\nabla \times \mathbf{E} & =i Z_{0} k_{0} \mathbf{H}, \\
\nabla \times \mathbf{H} & =-i k_{0} / Z_{0} \mathbf{n}^{2} \cdot \mathbf{E},
\end{aligned}
$$

and the conjugated form for $\mathbf{E}^{\prime *}$ and $\mathbf{H}^{\prime *}$ :

$$
\begin{aligned}
& \nabla \times \mathbf{E}^{\prime *}=-i Z_{0} k_{0} \mathbf{H}^{\prime *}, \\
& \nabla \times \mathbf{H}^{\prime *}=i k_{0} / Z_{0} \mathbf{n}^{\prime 2} \cdot \mathbf{E}^{\prime *},
\end{aligned}
$$

where $k_{0}=2 \pi / \lambda$ is the free-space wave number and $Z_{0}=\sqrt{\mu_{0} / \varepsilon_{0}}=377 \Omega$ is the electromagnetic impedance in vacuum, we obtain the following reciprocal formula for propagation constants:

$$
\begin{aligned}
\beta_{j}=\beta_{k}^{\prime}+\frac{k_{0}}{Z_{0}} \frac{\int_{A_{\infty}} \mathbf{e}_{j} \cdot\left(\mathbf{n}^{2}-\mathbf{n}^{\prime 2}\right) \cdot \mathbf{e}_{k}^{\prime *} \mathrm{~d} A}{\int_{A_{\infty}}\left(\mathbf{e}_{j} \times \mathbf{h}_{k}^{\prime *}+\mathbf{e}_{k}^{\prime *} \times \mathbf{h}_{j}\right) \cdot \mathbf{z} \mathrm{d} A} \\
=\beta_{k}^{\prime}+\frac{k_{0}}{Z_{0}} \frac{\int_{A_{\infty}}\left(\delta n_{t}^{2} \mathbf{e}_{t j} \cdot \mathbf{e}_{t k}^{\prime}+\delta n_{z}{ }^{2} e_{z j} e_{z k}^{\prime}{ }^{*}\right) \mathrm{d} A}{\int_{A_{\infty}}\left(\mathbf{e}_{j} \times \mathbf{h}_{k}^{\prime *}+\mathbf{e}_{k}^{*} \times \mathbf{h}_{j}\right) \cdot \mathbf{z d} A} .
\end{aligned}
$$

Here we define $\delta n_{t}{ }^{2}=n_{t}{ }^{2}-n_{t}{ }^{2}$ and $\delta n_{z}{ }^{2}=n_{z}{ }^{2}-n_{z}{ }^{2}$. With the aid of the reciprocal formula we can expand the transverse mode fields $\left(\mathbf{e}_{t j}, \mathbf{h}_{t j}\right)$ in terms of the primed ones:

$$
\begin{aligned}
\mathbf{e}_{t j} & =\sum_{k}\left(a_{j k}+b_{j k}\right) \mathbf{e}_{t k}^{\prime}, \\
\mathbf{h}_{t j} & =\sum_{k}\left(a_{j k}-b_{j k}\right) \mathbf{h}_{t k}^{\prime} .
\end{aligned}
$$

We can find the expansion of the longitudinal fields by substituting Eqs. (A10) into Maxwell's equations (A7) and (A8):

$$
\begin{aligned}
& e_{z j}=\frac{n_{z}^{\prime 2}}{n_{z}^{2}} \sum_{k}\left(a_{j k}-b_{j k}\right) e_{z k}^{\prime}, \\
& h_{z j}=\sum_{k}\left(a_{j k}+b_{j k}\right) h_{z k}^{\prime} .
\end{aligned}
$$

Substituting Eqs. (A10) into reciprocal formula (A9) and using the orthogonality condition, we find the following expansion coefficients $a_{j k}$ and $b_{j k}$ :

$$
\begin{aligned}
a_{j k} & =\frac{k_{0}}{4\left(\beta_{j}-\beta_{k}^{\prime}\right)} \frac{1}{Z_{0}} \int_{A_{\infty}}\left(\delta n_{t}^{2} \mathbf{e}_{t j} \cdot \mathbf{e}_{t k}{ }^{\prime *}+\delta n_{z}{ }^{2} e_{z j} e_{z k}^{\prime}{ }^{*}\right) \mathrm{d} A, \\
b_{j k} & =\frac{k_{0}}{4\left(\beta_{j}+\beta_{k}^{\prime}\right)} \frac{1}{Z_{0}} \int_{A_{\infty}}\left(\delta n_{t}{ }^{2} \mathbf{e}_{t j} \cdot \mathbf{e}_{t k}{ }^{*}-\delta n_{z}{ }^{2} e_{z j} e_{z k}^{\prime} *\right) \mathrm{d} A .
\end{aligned}
$$

The authors are grateful for partial support by the National Science Council, Taiwan, under contract NSC 89-2215-E-002-013 and by the Education Ministry of Taiwan under contract 89-E-FA06-2-4.

\section{References}

1. A. M. Vengsarkar, P. J. Lemaire, J. B. Judkins, V. Bhatia, T. Erdogan, and J. E. Sipe, "Long-period fiber gratings as bandrejection filters," J. Lightwave Technol. 14, 58-65 (1996).

2. A. M. Vengsarkar, J. R. Pedrazzani, J. B. Judkins, P. J. Lemaire, N. S. Bergano, and C. R. Davidson, "Long-period fibergrating-based gain equalizers," Opt. Lett. 21, 336-338 (1996).

3. B. Ortega, L. Dong, W. F. Liu, J. P. de Sandro, L. Reekie, S. I. Tsypina, V. N. Bagratashvili, and R. I. Laming, "Highperformance optical fiber polarizers based on long-period gratings in birefringent optical fibers," IEEE Photon. Technol. Lett. 9, 1370-1372 (1997).

4. V. Bhatia and A. M. Vengsarkar, "Optical fiber long-period grating sensors," Opt. Lett. 21, 692-694 (1996).

5. H. J. Patrick, G. M. Williams, A. D. Kersey, J. R. Pedrazzani, and A. M. Vengsarkar, "Hybrid fiber Bragg grating/long period fiber grating sensor for strain/temperature discrimination," IEEE Photon. Technol. Lett. 8, 1223-1225 (1996).

6. A. A. Abramov, B. J. Eggleton, J. A. Rogers, R. P. Espindola, A. Hale, R. S. Windeler, and T. A. Strasser, "Electrically tunable efficient broad-band fiber filter," IEEE Photon. Technol. Lett. 11, 445-447 (1999).

7. H. S. Kim, S. H. Yun, I. K. Kwang, and B. Y. Kim, "All-fiber acousto-optic tunable notch filter with electronically controllable spectral profile," Opt. Lett. 22, 1476-1478 (1997).

8. C. Y. Lin and L. A. Wang, "Loss-tunable long period fibre grating made from etched corrugated structure," Electron. Lett. 35, 1872-1873 (1999).

9. T. Erdogan, "Cladding-mode resonances in short- and longperiod fiber grating filters," J. Opt. Soc. Am. A 14, 1760-1773 (1997).

10. G. W. Chern and L. A. Wang, "Transfer-matrix method based on perturbation expansion for periodic and quasi-periodic binary long-period gratings," J. Opt. Soc. Am. A 16, 2675-2689 (1999).

11. M. Song, B. Lee, S. B. Lee, and S. S. Choi, "Interferometric temperature-insensitive strain measurement with differentdiameter fiber Bragg gratings," Opt. Lett. 22, 790-792 (1997).

12. J. F. Nye, Physical Properties of Crystals (Clarendon, Oxford, 1969).

13. A. W. Snyder and J. D. Love, Optical Waveguide Theory (Chapman \& Hall, London, 1991), Secs. 31-1 and 31-8.

14. A. Wexler, "Solution of waveguide discontinuities by modal analysis," IEEE Trans. Microwave Theory Tech. MTT-15, 508-517 (1967).

15. R. Kuszelewicz and G. Aubert, "Modal matrix theory for light propagation in laterally restricted stratified media," J. Opt. Soc. Am. A 14, 3262-3272 (1997).

16. C. Dragone, "Scattering at a junction of two waveguides with different surfaces impedences," IEEE Trans. Microwave Theory Tech. MTT-32, 1319-1328 (1984).

17. W.-P. Huang and J. Hong, "A transfer matrix approach based on local normal modes for coupled waveguides with periodic perturbation," J. Lightwave Technol. 11, 1367-1374 (1992).

18. M. N. O. Sadiku, Numerical Techniques in Electromagnetics (CRC Press, Boca Raton, Fla., 1992).

19. T. W. MacDougall, S. Pilevar, C. W. Haggans, and M. A. Jackson, "Generalized expression for the growth of long period gratings," IEEE Photon. Technol. Lett. 10, 1449-1451 (1998). 